Document downloaded from:

http://hdl.handle.net/10251/38950

This paper must be cited as:

Molina Mateo, J.; Torregrosa Cabanilles, C.; Sabater I Serra, R.; Meseguer Dueñas, JM.; Gómez Ribelles, JL. (2013). Computer simulation of the heterogeneity of segmental dynamics inamorphous polymers. Journal of Non-Crystalline Solids. 362:175-179. doi:10.1016/j.jnoncrysol.2012.11.034.

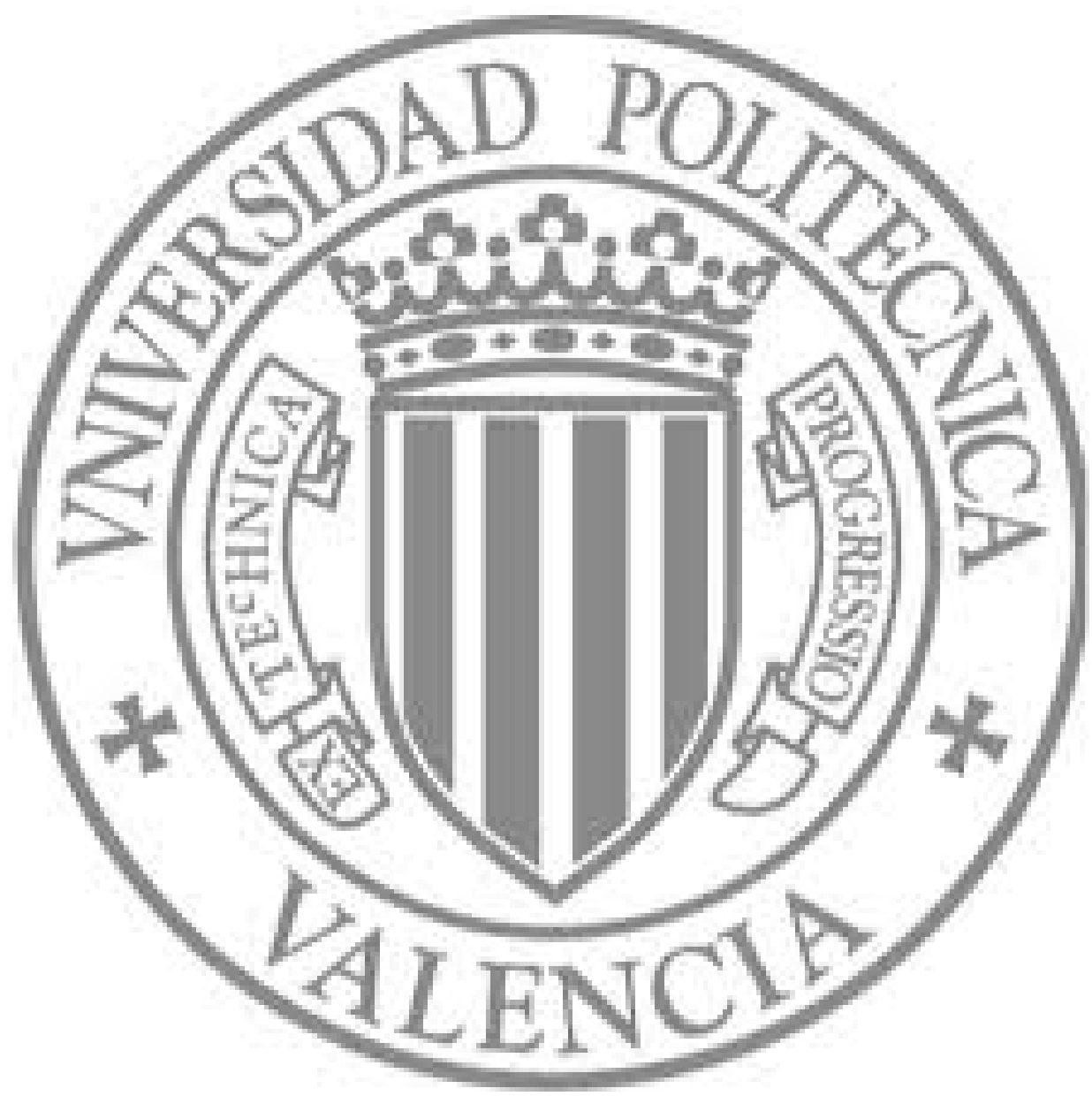

The final publication is available at

http://dx.doi.org/10.1016/j.jnoncrysol.2012.11.034

Copyright Elsevier 


\title{
Computer simulation of the heterogeneity of segmental dynamics in amorphous polymers
}

J. Molina-Mateo ${ }^{1}$, C. Torregrosa-Cabanilles ${ }^{1}$, R. Sabater-Serra ${ }^{1}$, J.M. MeseguerDueñas $^{1,2}$, J.L. Gómez-Ribelles ${ }^{1,2,3}$

${ }^{1}$ Center for Biomaterials and Tissue Engineering, Universitat Politècnica de València, Camino de Vera s/n, 46022 Valencia, Spain

${ }^{2}$ Networking Research Center on Bioengineering, Biomaterials and Nanomedicine (CIBER-BBN), Valencia, Spain

${ }^{3}$ Prince Felipe Research Center, Autopista del Saler 16, 46013 Valencia, Spain

\begin{abstract}
The heterogeneity of local segmental dynamics in a polymer system was analysed by computer simulation with the Bond Fluctuation Model. In a previous work we showed the difficulties encountered in characterizing this heterogeneity by means of a distribution of relaxation times. In this work a different approach is proposed based on the concept of Dynamically Accessible Volume (DAV). A DAV value was assigned to each polymer chain as the fraction of cells in its surroundings that could be occupied in one Monte Carlo step. In this way it was possible to relate the mobility of a chain with the accessible volume around it, due to the relationship between DAV and diffusion coefficient. As temperature decreases in equilibrium the DAV distribution shifts towards lower values, its width decreases and the number of frozen molecules increases. The methodology proposed also provides a way of characterizing the evolution of segmental dynamics distribution in out of equilibrium states below glass transition temperatures.
\end{abstract}

\section{Keywords}

Dynamically Accessible Volume, Bond Fluctuation Model, Relaxation Times 


\section{1.-Introduction}

Polymeric materials in liquid and glassy states have physical properties that are unique with respect to other materials, including: $700 \%$ elastic deformations, change of mechanical response rate in several orders of magnitude with small changes in temperature, memory effect, a swelling capacity in certain solvents of several hundred per cent without dissolving and many others. As is well known, the key feature behind these particular properties is their special molecular mobility. Conformational rearrangements of the main chains in the equilibrium liquid or rubber-like state allow deformation of the polymer chain coils to such an extent that the above-mentioned macroscopic deformation occurs. The response time, which can be characterized by an average relaxation time, depends exponentially on temperature, in accordance with a law that can be described by the Williams-Landel-Ferry (WLF) or Vogel-FulcherTamman-Hesse (VFTH) equations. The representation of the logarithm of the relaxation time against reciprocal temperature shows a characteristic curvature with two asymptotes. At high temperatures the relaxation times tend to the reciprocal of natural vibration frequencies, around -14 [1,2] while they tend to infinity around 50 degrees below the glass transition temperature $T_{g}$, at which temperatures the high slope of the $\log \tau$ vs. 1/T plot means that a 3 -degree temperature change produces a change in the order of a decade in the relaxation time [3]. The exponential dependence of mobility on temperature is quite intuitive and common to other thermally activated phenomena. However, the special conformational mobility of polymers (and low molecular weight glass-formers) is that relaxation times also depend exponentially on the packing density of molecules or polymer segments. This means that, at a given temperature, the relaxation time in a microscopic region of the material could be more than 6 orders of magnitude longer than other regions, due to the spatial heterogeneity of the material. Relatively small density fluctuations across the material volume yield considerable heterogeneity of the local dynamic response. This feature has been characterized by distributions of relaxation times since the first studies on relaxation processes in polymeric materials [3-5].

Broad asymmetric distributions of relaxation times have been determined for the co-operative conformational motions of polymer chain segments from studies of viscoelastic or dielectric main or alpha relaxation that takes place at temperatures 
immediately above the glass transition or from studies of the kinetics of structural relaxation. The heterogeneity of conformational mobility at temperatures around and below the glass transition region is another interesting phenomenon. A glass-forming material, in particular an amorphous polymer in a glassy state, at temperatures below or in the range of the glass transition is in a non-equilibrium state with higher specific volume, enthalpy and entropy than those of thermodynamical equilibrium. Under constant environmental conditions it evolves into an equilibrium state, a process known as structural relaxation or physical ageing. This process involves the close packing of the molecules or chain segments by means of conformational rearrangements. During structural relaxation, specific volume continuously decreases and, as a consequence, relaxation times increase. An interesting and open question is how local dynamics heterogeneity evolves during this isothermal process. One might hypothesize that the regions with the longest relaxation times, i.e., with the most closely packed molecules could collapse at the beginning of the process and undergo no further evolution, while the more loosely packed regions would be responsible for contraction and consequently become slower and slower. The shape of the relaxation time distribution could then be expected to evolve. However, the contrary can also be assumed: the evolution of the relaxation time of each individual microscopic region during structural relaxation would be parallel to each other in the logarithm of the relaxation time plot, leaving the distribution of relaxation times unchanged. The latter case has been assumed by most structural relaxation phenomenological models.

Experimental research in this subject is hindered by the fact that the experimental time necessary to reach conclusions only a few degrees below the glass transition becomes unrealistic and also because the distribution of relaxation times is not a measurable magnitude but is calculated from the time evolution of physical variables, a calculation that needs the hypothesis that it is intended to prove.

In this work we studied this problem using the Bond Fluctuation Model, BFM, a coarse-grained Monte-Carlo model that has proved capable of reproducing some essential features of glass transition and structural relaxation in previous studies [6]. The distribution of relaxation times was determined by analysing segmental self-diffusion in a number of small regions of the model lattice, as explained below. These distributions have been calculated in other studies by means of Molecular Dynamics $[7,8]$, but to our 
knowledge this is the first time that this problem has been analyzed by the BFM. Model simulations showed that the distribution shifted toward longer times as the temperature decreased while broadening [9]. Nevertheless, due to the definition of the relaxation time in Monte-Carlo simulation [9], the simulation time required to calculate the relaxation time is in the order of the relaxation time itself and thus grows as temperature decreases. This limits calculations to temperatures above the glass transition.

In this work we aim at a different approach and try to analyse the relationship between free volume distribution (or static heterogeneity) and dynamic heterogeneity, which will be characterized through the dynamic accessibility of the available free volume.

\section{2.-Simulations}

The Bond Fluctuation Model is a Monte Carlo simulation model that reproduces the main features of polymeric materials [10-15]. It consists of a cubic lattice in which molecular groups that represent segments of the main chain occupy the empty space forming cubes. These molecular groups are bonded to form the polymeric material and the distance allowed for the bonds can fluctuate between 2 and $\sqrt{10}$ lattice units. The dynamics of the model consist of randomly choosing a molecular group and a direction of movement. The movement is finally performed with a probability that is given by the Metropolis criterion [12]:

$$
P=\min \left[1, \exp \left(\frac{-\Delta E}{k_{b} T}\right)\right]
$$

This expression takes into account the variation of energy $\Delta E$ caused by the evaluated movement and temperature: $k_{b}$ is Boltzmann's constant and $\mathrm{T}$ is the temperature of the system. In this work we employed two potentials in order to represent the energy. First, a Lennard-Jones potential calculated for all non-bonded monomers up to a distance of four lattice units [11]:

$$
U_{L J}=4 \varepsilon\left[\left(\frac{\sigma}{r}\right)^{12}-\left(\frac{\sigma}{r}\right)^{6}\right]
$$


where $\sigma$ was 2.0 , in order to be consistent with the excluded volume imposed by the lattice conditions of the Bond Fluctuation Model. The second was a bond length potential [11]:

$$
U(l)=U_{0}\left(l-l_{0}\right)^{2}
$$

in which the minimal energy distance was $1_{0}=3.0$ lattice units.

In our previous work [9] we followed the dynamics of each polymer chain through the time correlation function of its center of mass, whose position is defined by $\vec{r}_{c m}$ :

$$
g_{3}(t)=\left\langle\left[\vec{r}_{c m}(t)-\vec{r}_{c m}(0)\right]^{2}\right\rangle
$$

The relaxation time of the chain is the time at which $g_{3}(t)$ equals the squared radius of gyration [11]:

$$
\left\langle R_{g}^{2}(N)\right\rangle=\frac{1}{N}\left\langle\sum_{i=1}^{N}\left|\vec{r}_{i}-\vec{r}_{c m}\right|^{2}\right\rangle
$$

where $N$ is the number of monomeric groups in the chains, and $\vec{r}_{i}$ is the position of each group in the chain. Thus, if $\tau$ is the relaxation time

$$
g_{3}(\tau)=\left\langle R_{g}^{2}\right\rangle
$$

In [9] all the relaxation times $\tau$ were calculated simultaneously for all the polymer chains, taking into account their own mobility. These calculations made it possible to build the shape of the distribution of the relaxation times, obtaining a more accurate representation of the evolution of the system as a function of time.

Nevertheless, this characterization of the heterogeneity of local dynamics presents certain problems inherent in relaxation time calculations. The method was limited to high temperatures as the simulation time required to calculate $\tau$ in Equation 6 was as long as the relaxation time itself, so that simulation time increased dramatically as temperature decreased. Furthermore, the definition was not appropriate when the 
system was out of equilibrium, since the radius of gyration changes during the calculations due to the relaxation process. In fact, the method required more simulation time for out of equilibrium situations than the process that we wanted to observe and the results were not reliable.

We propose in this work another approach based on the concept of Dynamically Accessible Volume (DAV) [16-18] and its formulation for thermal systems [19]. DAV calculates the probability (taking into account the Metropolis criterion) of every empty cell being occupied in one Monte Carlo Step and labels the empty space in the simulation box with a probabilistic map of occupation. The average value of this probability provides a measure of the available empty space in the system that can contribute to the diffusion of the molecules. The DAV calculates the fraction of cells that can be occupied in one Monte Carlo Step, contributing to the diffusion of the system:

$$
D A V=\frac{1}{L^{3}} \sum_{j=1}^{L^{3}}\left\{\min \left[1, \sum_{1=1}^{n n} \exp \left(\frac{-\Delta E_{i j}}{k_{B} T}\right)\right]\right\}
$$

$L$ is the box simulation size, $n n$ the number of neighbours a cell has and $\Delta E_{i j}$ is the variation in the energy of the system caused by a movement of a monomer from cell $i$ to cell $j$. This parameter offers a landscape of the available space in the system that aids in diffusion. We associate each empty accessible cell with the polymer chain that can occupy it in one Monte-Carlo step and thus, a DAV value can be assigned to each polymer chain as the fraction of the cells in its surroundings that can be occupied. The rationale for this treatment is to relate the mobility of a chain with the accessible volume around it due to the relationship between DAV and the diffusion coefficient [20]. A DAV distribution arises due to the different neighbourhoods of the different chains imposed for local molecular packing. A histogram is built in 0.025 DAV units step and the distribution, normalized to the number of chains in the system, is calculated from the histogram. The main advantage of this approach is that the DAV distribution can be calculated from a static image of the system, so that long simulations are not needed to build the distribution. Furthermore, this method can be applied to systems that relax out of equilibrium, monitoring the dynamics of the system during the process. 
In order to validate the concept we performed simulations in a $\mathrm{L}=40$ lattice with periodic boundary conditions, occupied by chains consisting of ten molecular groups. Density was $\phi=0.5$, so the system consisted of 400 chains. Energy parameters were $\varepsilon=0.25, \mathrm{U}_{0}=0.75, \sigma=2.0$ and $\mathrm{l}_{0}=3.0$. Simulation with these parameters have shown [21] a glass transition in cooling ramps and structural relaxation in isothermal annealing below the glass transition interval. Every simulation was repeated 20 times in order to obtain reliable data.

The thermal history began with an initial equilibration period of $10^{5}$ Monte Carlo Steps (MCS) at $k_{b} T=5$, which led to a liquid in equilibrium. After this initial period, a cooling rate of 0.1 units of $k_{b} T$ per every 1000 MCS was applied until reaching different temperatures, followed by isothermal annealing for $10^{7} \mathrm{MCS}$. The DAV distribution was calculated at different times during the isotherms and at different temperatures throughout the cooling ramp between $k_{b} T=5$ and $k_{b} T=0.01$.

\section{3.-Results and discussion}

Energy and DAV evolution during the cooling ramp are shown in Figures 1 and 2, respectively. Both show the equilibrium liquid line at high temperatures, with decreasing energy and accessible free volume as temperature decreases. The departure from equilibrium starts around $k_{B} T=0.4$ and gradually changes to the glassy state, characterized by a temperature-independent energy value and DAV values almost zero, which highlights the lack of mobility of the system at low temperatures in the glassy state. The extrapolation of the equilibrium liquid line in the DAV against $1 / k_{B} T$ plot allows the definition of the glass transition temperature from its intersection with the $\mathrm{DAV}=0$ axis. This is the same value obtained by extrapolation of liquid and glass lines in the energy plot. In this simulation we obtain $k_{B} T_{g}$ around 0.25 . In order to define the temperature interval of the glass transition more precisely, simulations of isothermal annealing were performed at different temperatures. The first temperature at which evolution of total energy or average DAV was detected was $k_{B} T=0.35$. At higher temperatures the system must be considered in equilibrium in the state reached on cooling. At the lowest temperatures no DAV or energy evolution is detected because of the extreme lack of mobility of the frozen chains. We can arbitrarily fix the lowest limit 
of the glass transition when no changes in DAV are detected after $10^{7}$ MonteCarlo steps. In this way the glass transition interval can be defined between $k_{B} T=0.35$ and a temperature around $k_{B} T=0.2$ when accessible volume is already nearly zero.

Figures 3 to 5 present the DAV distribution for a set of selected temperatures, calculated for the state attained by the system when reaching this temperature in the cooling ramp. It is worth noting that all curves had the same area, since all simulations had the same number of molecules. This highlights the fact that the height of the distribution also characterizes its width: an increase in the peak height also implies that the distributions become narrower, which makes it easier to follow the evolution of the DAV distribution. The inset in Figure 3, shows the ratio of totally blocked molecules $(\mathrm{DAV}=0)$ according to the reciprocal temperature. The number of blocked molecules was representative of the transition that was previously shown in Figures 1 and 2 and highlights the lack of available volume as the driving force of the transition.

Since the DAV value is calculated as a fraction of the total cells, the figures calculated for the total system and shown in Figure 2 can be compared with those appearing in the abscissa of the plots representing the DAV distribution in Figures 3 to 6. At the highest equilibrium temperatures the distribution is more or less symmetrical for $k_{B} T=5$ (Figure 3 ) and roughly covers the DAV interval between 0 and 0.35 . This range is even larger than that covered by the average DAV value for the complete system when temperature changes from $k_{B} T=5$ to $k_{B} T=0.01$. This means that at this temperature there are some chains in the system whose neighbourhood is so dense that they lack any mobility, while others behave as if they were at the highest temperatures due to a very loose local chain packing. The most frequent DAV value, that of the peak of the distribution, is 0.175 (Figure 3).

As temperature decreases while the system is in equilibrium, for $0.4 \leq k_{B} T \leq 5$, the whole distribution shifts towards lower DAV values. The most frequent value moves from 0.175 to 0.05 when $k_{B} T$ changes from 5 to 0.4 . At the same time the distributions become narrower, the peaks increase and become slightly asymmetrical. It should be noted that, since the lower DAV limit is zero, after a certain temperature the distribution begins to accumulate at its lower limit. The value of the intersection of the distribution with the $\mathrm{DAV}=0$ axis gives a measurement of the number of fully frozen 
chains, i.e., chains in which all the segments have no available space in their neighbouring lattice sites to move in one Monte Carlo step. Since the plot of Figure 3 is a normalized histogram, it can be seen that even in equilibrium there is a fraction of frozen chains which can be up to $10 \%$ in the case of $k_{B} T=0.4$. The inset in Figure 3 shows the fraction of frozen chains as a function of temperature.

The distribution in the glass transition temperature range (thus both above and below the glass transition temperature $k_{B} T=0.25$ ) are presented in Figure 4. Interestingly, during transition the fraction of blocked chains increases rapidly, as shown in the inset of Figure 3. However, the right hand side of the distribution continues shifting towards lower DAV values, as expected. If we take the glass transition temperature as reference, at this temperature the maximum of the distribution is nearly zero and roughly speaking no chain has an accessible volume in its neighbourhood above 0.15. At lower temperatures the DAV distribution is a monotonously decreasing function of DAV. In this range of temperatures most, though not all, of the chains are frozen and structural relaxation can therefore take place if the system is maintained in isothermal conditions.

At temperatures below the glass transition range, for $k_{B} T \leq 0.2$ the mobility of the system is very restricted, with a fraction of fully frozen chains above 60\% (which increases to $100 \%$ at $k_{B} T=0.05$ ) and maximum DAV values of 0.075 . This explains why structural relaxation cannot be observed in our $10^{7}$ Monte Carlo steps simulation.

The final goal of the work was to determine the effect of structural relaxation on chain mobility. As mentioned in the introduction, the characterization of chain mobility in out of equilibrium states by means of a distribution of relaxation times has serious limitations. In our previous work [9] we showed that the simulation time needed to build the distribution of the relaxation was so long that it interferes with the structural relaxation itself. On the other hand, on the right hand side of the distribution there appear a number of chains that move too fast for the simulation time required to evaluate their relaxation time. The situation is different if the characterization of chain mobility is performed in terms of free volume availability in its neighbouring lattice sites. In this way we take a picture of the lattice at a given time and calculate the probability of each empty site being occupied to obtain the DAV per molecule. 
The change in the DAV distribution after $10^{7}$ MCS is shown in Figure 6 for $k_{B} T=0.35,0.3$ and 0.2 . At $k_{B} T=0.35$ (Figure 6a), the highest temperature at which structural relaxation takes place, the distribution shifts towards lower DAV values while the height of the peak increases and the fraction of fully frozen chains increases significantly, from around 0.15 to 0.25 . At $k_{B} T=0.3$ (Figure 6b) the evolution during isothermal annealing is clear: the fraction of frozen chains increases from 0.25 to more than 0.55 and there are no chains with local DAV values above 0.1. At $k_{B} T=0.2$ (Figure 6c) mobility is completely lost after the isothermal annealing.

\section{4.-Conclusions}

The DAV distribution characterizes the chain mobility of a system calculated from a static image and can offer a landscape of the dynamics of the system. This approach shows that at any given time, even at temperatures well above the glass transition, part of the polymer chains have no free volume around them in which to move, while others move as freely as they would do at the highest temperatures. The fraction of frozen chains rapidly increases when the behaviour of the system separates from the equilibrium state in the glass transition. The fact that mobility is deduced from an instantaneous image of the system structure means that the evolution of the material can be studied out of equilibrium. Structural relaxation in the glass transition range makes the distribution shift towards lower DAV values but mainly increases the fraction of chains without any mobility.

\section{Acknolwedgements}

The authors would like to acknowledge the support provided by the Conselleria d'Educació of the Generalitat Valenciana through the GV/2009/033 project. The translation of this paper was funded by the Universidad Politécnica de Valencia, Spain.

\section{References}

[1] Donth E. Relaxation and Thermodynamics in Polymers, Glass Transition. Berlin: Akademie Verlag, 1992.

[2] Angell CA. J. Non-Cryst. Solids 1991;13:131-133. 
[3] Ferry JD. Viscoelastic Properties of Polymers, 3rd ed. New York: John Wiley \& Sons, 1980.

[4] McCrum NG, Read BE, Williams G. Anelastic and Dielectric Effects in Polymeric Solids. New York: Dover Publications Inc., 1967.

[5] Kremer F, Schönhals A. Broadband Dielectric Spectroscopy. Berlin, Heidelberg: Springer-Verlag, 2003.

[6] Arnoult M, Saiter JM, Pareige C, Meseguer-Dueñas JM, Gómez-Ribelles JL, Molina-Mateo J. The Journal of Chemical Physics 2009;30:214905.

[7] Ahumada O, Theodorou DN, Triolo A, Arrighi V., Karatasos C, Ryckaert JP. Macromolecules 2002;35:7110-7124.

[8] Peter S, Meyer H, Baschnagel J. The European Physical Journal E 2009;28:147-158.

[9] Molina-Mateo J, Meseguer-Dueñas JM, Gómez-Ribelles JL, Torregrosa-Cabanilles C. Polymer 2009;50:5618-5622.

[10] Carmesin I, Kremer K. Macromolecules 1988;21:2819-2823.

[11] Binder K. Monte Carlo and molecular dynamics simulations in polymer science. Oxford: Oxford University Press, 1995.

[12] Deutsch H, Binder K. Journal of Chemichal Physics 1990;94:2294-2304.

[13] Paul W, Binder K, Heermann D, Kremer K. Journal of Chemichal Physics 1991; 95(10):7726-7740.

[14] Lobe B, Baschnagel J, Binder K. Macromolecules 1994;27:3658-3665.

[15] Di Cecca A, Freire JJ. Macromolecules 2002;35:2851-2858.

[16] Dawson KA, Lawlor A, McCullagh GD, Zaccarelli E, Tartaglia P. Physica A 2002; 316:115-134.

[17] Dawson KA, Lawlor A, DeGregorio P, McCullagh GD, Zaccarelli E, Fo G, Tartaglia P. Faraday Discussions 2003;123:13-26.

[18] Lawlor A, Reagan D, McCullagh GD, De Gregorio P, Tartaglia P, Dawson K. Physical Review Letters 2002;89(24):2455031-2455034.

[19] Molina-Mateo J, Meseguer-Dueñas JM, Gómez-Ribelles JL. Macromolecular Theory and Simulations 2006;15:32-39.

[20] Molina-Mateo J, Meseguer-Dueñas JM, Gómez-Ribelles JL, Polymer 2006;47: 4861-4865.

[21] Arnoult M, Saiter JM, Pareige C, Meseguer-Dueñas JM, Gómez-Ribelles JL, Molina-Mateo J. The Journal of Chemical Physics 2009;130:214905. 
Figure 1: Energy per molecular group (mg) according to the reciprocal of temperature during the cooling ramp. Lines are shown only as a guide.

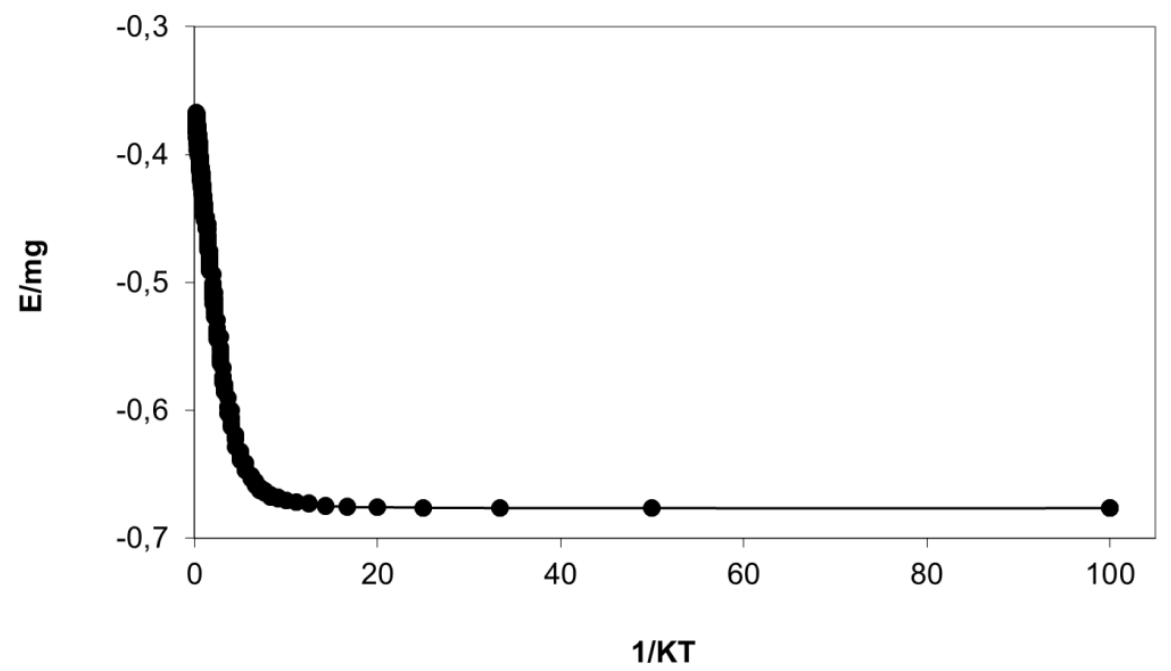


Figure 2: Total Dynamically Accessible Volume of the system according to the reciprocal of temperature during the cooling ramp. Lines are shown only as a guide.

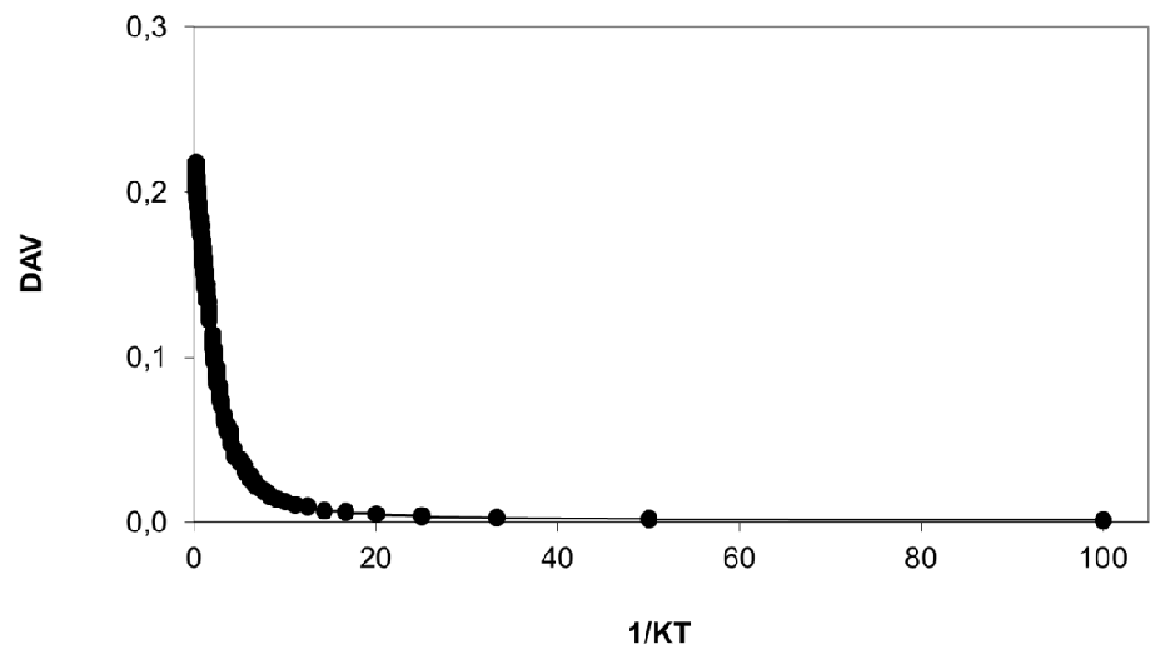


Figure 3: DAV distribution for different equilibrium temperatures during the cooling ramp (from right to left $k_{B} T=3, k_{B} T=0.9, k_{B} T=0.7, k_{B} T=0.5$ and $k_{B} T=0.4$ ). The inset shows the ratio of totally blocked molecules $(\mathrm{DAV}=0)$ according to the reciprocal temperature during the cooling ramp. Lines are shown only as a guide.

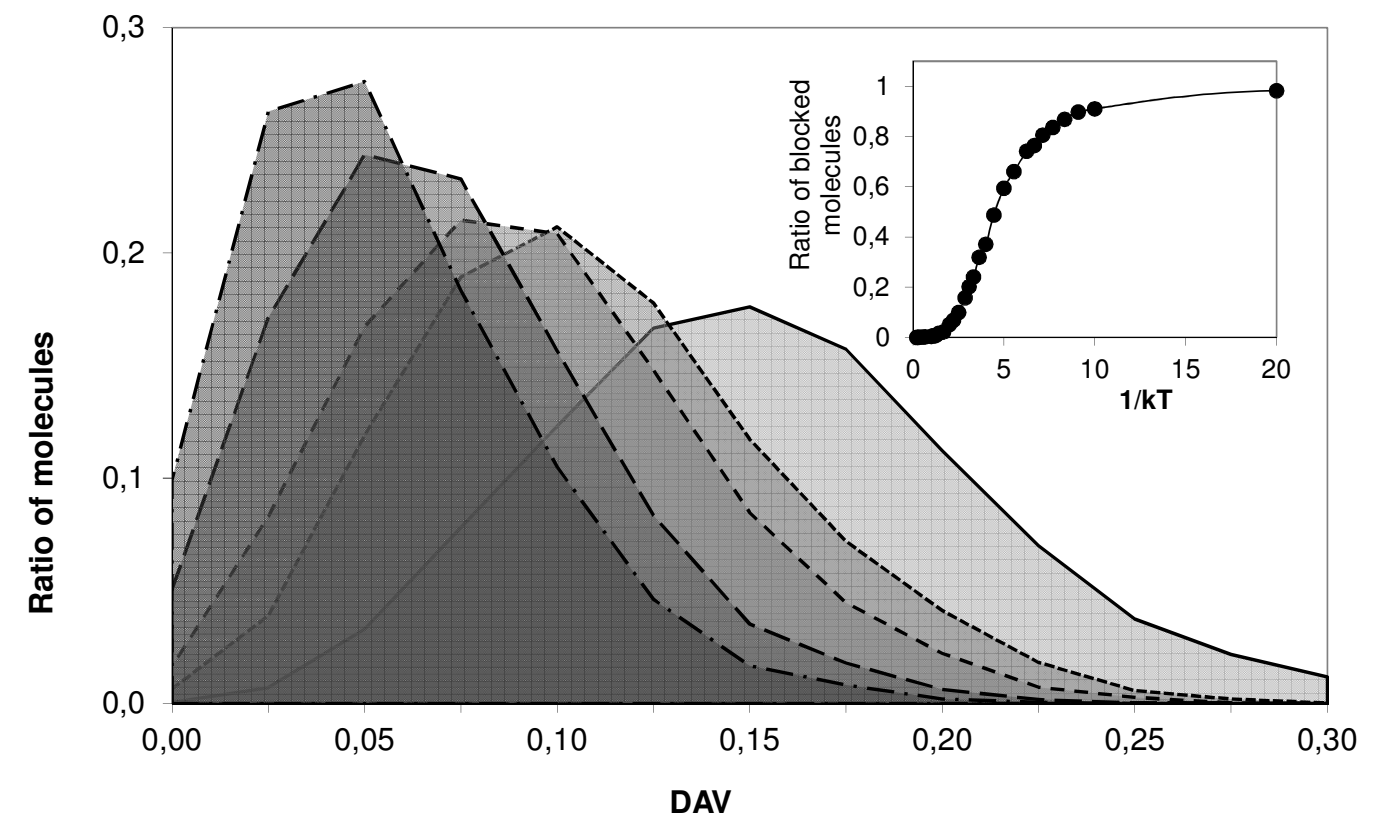


Figure 4: The DAV distribution for different temperatures in the glass transition region during the cooling ramp (from right to left $k_{B} T=0.4, k_{B} T=0.35, k_{B} T=0.3, k_{B} T=0.25$ and $\left.k_{B} T=0.2\right)$. Lines are shown only as a guide.

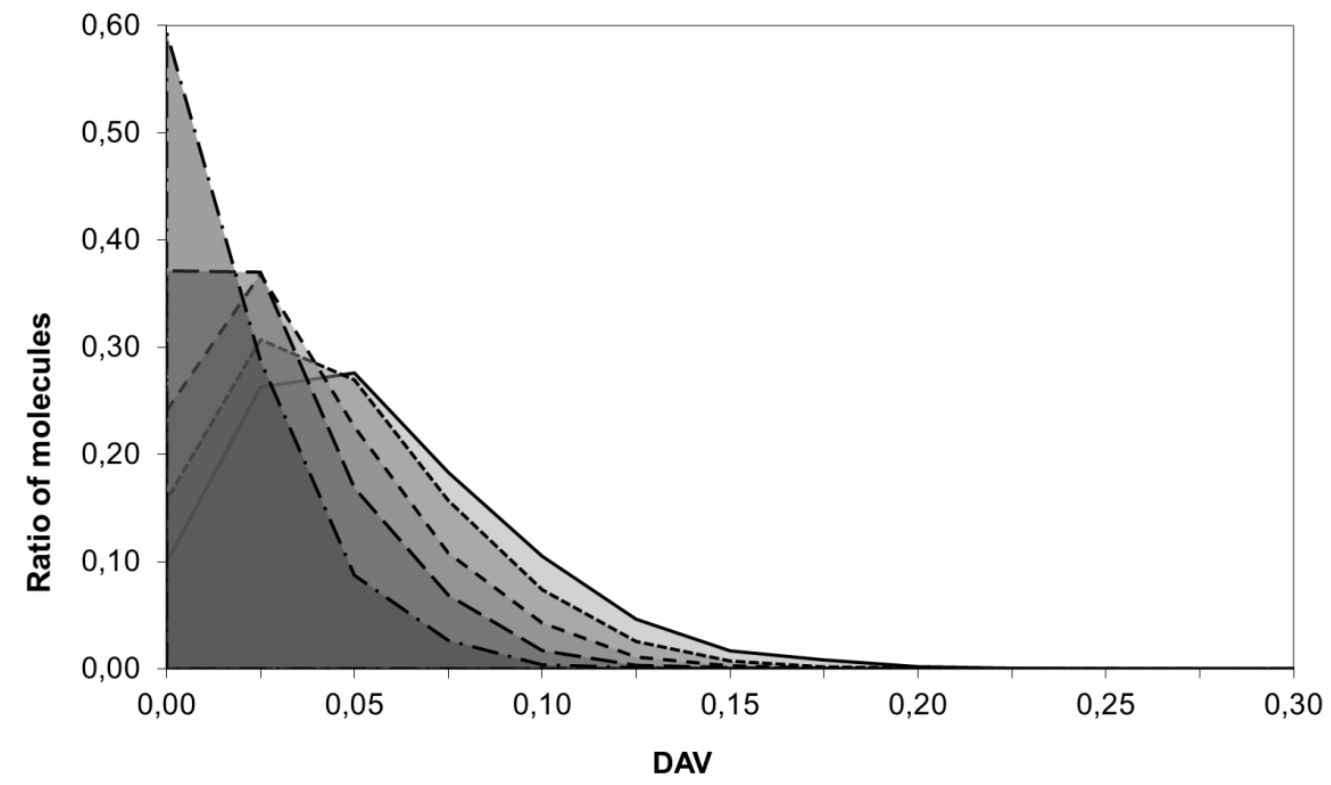


Figure 5: The DAV distribution for different temperatures below the glass transition region during the cooling ramp (from right to left $k_{B} T=0.2, k_{B} T=0.16, k_{B} T=0.14$, $k_{B} T=0.12, k_{B} T=0.1$ and $\left.k_{B} T=0.01\right)$. Lines are shown only as a guide.

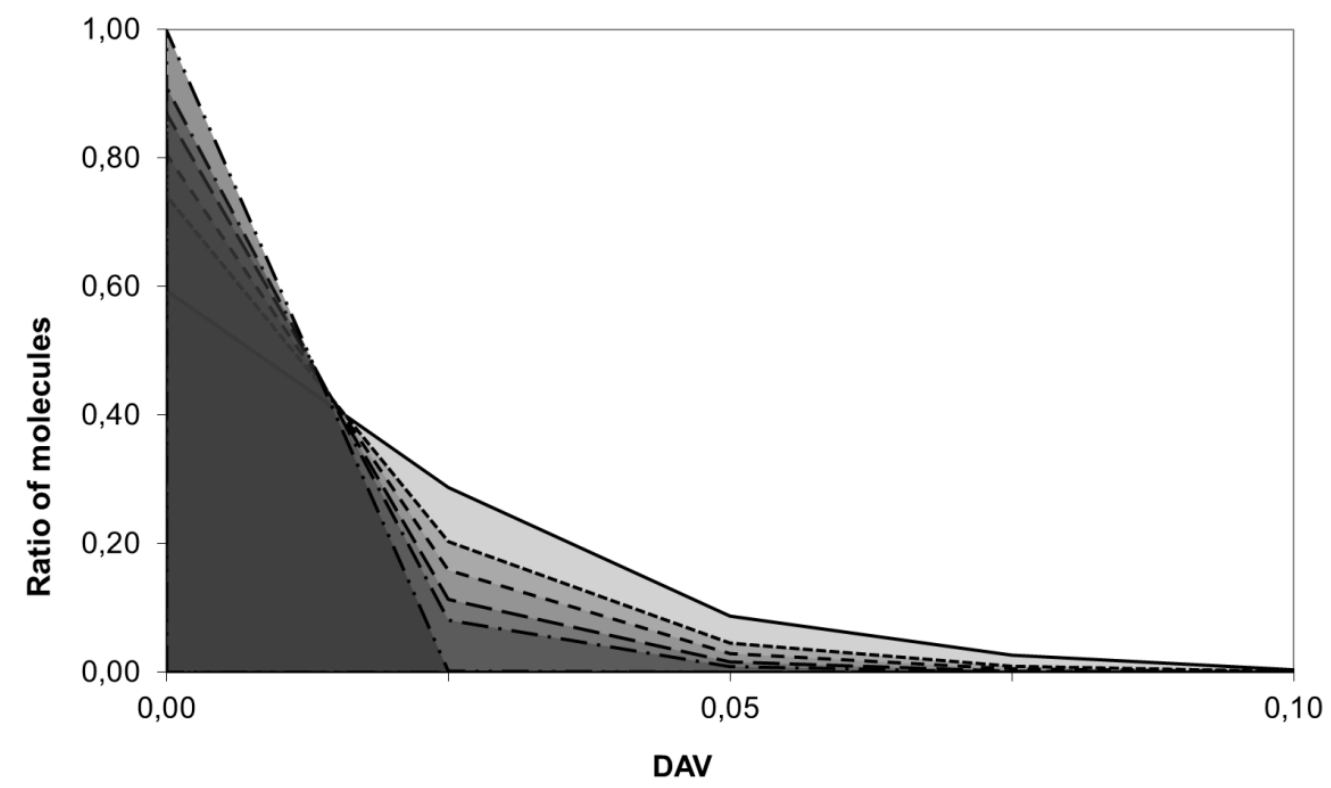


Figure 6: The DAV distribution before (continuous line) and after (dotted line) annealing at $k_{B} T=0.35$ (a), $k_{B} T=0.3$ (b) and $k_{B} T=0.2$ (c). Lines are shown only as a guide.
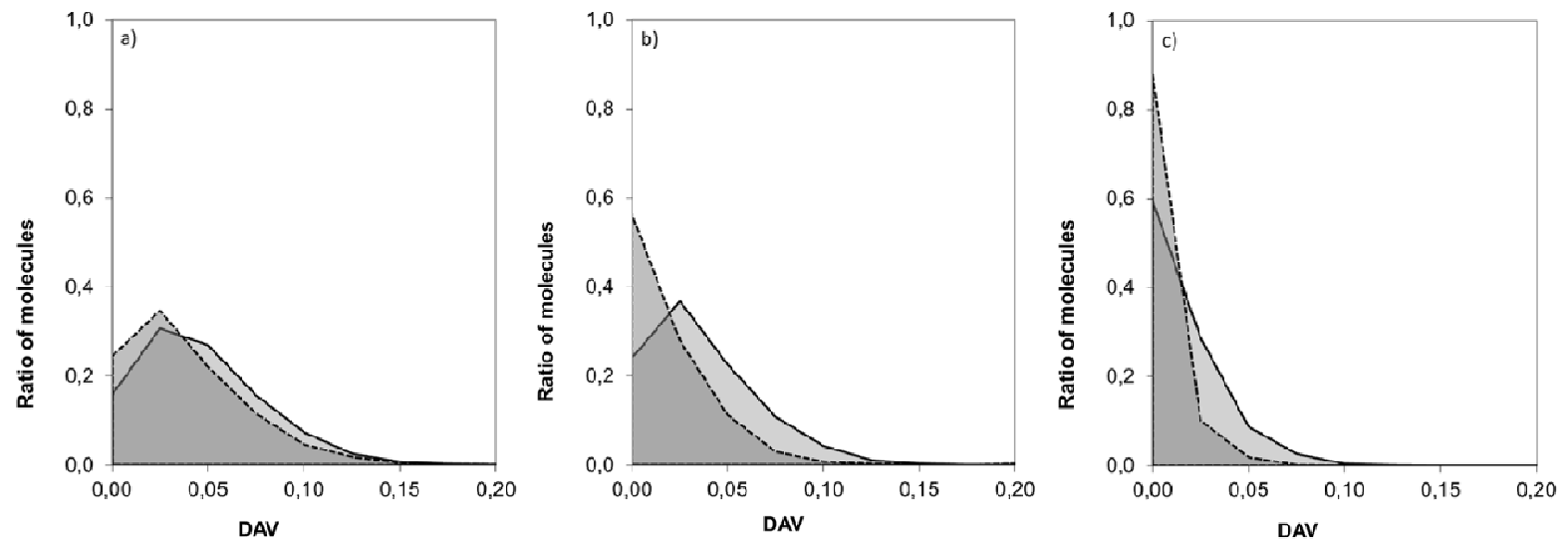